\title{
Gamificación en educación: una panorámica sobre el estado de la cuestión
}

\author{
Ana-M. Ortiz-Colón ${ }^{1}$ \\ Juan Jordán ${ }^{2}$ \\ Míriam Agredal ${ }^{1}$
}

\section{Resumen}

La sociedad del conocimiento y la tecnología han traído consigo un nuevo mapa en el que los jóvenes sienten inquietudes que la educación no siempre ha sabido satisfacer. Estos nuevos escenarios hacen que los intereses de los alumnos cambien, por lo que los profesores necesitan explorar nuevas estrategias y recursos en sus clases para aumentar la motivación y el compromiso con sus alumnos. El objetivo de este trabajo es hacer una revisión teórica de los beneficios del uso de la gamificación y conocer su aplicación en el contexto educativo. Para ello, se han examinado diversas publicaciones académicas provenientes de bases de datos internacionales presentadas entre 2011 y 2016, relacionadas con la aplicación de la gamificación en educación, la motivación e inmersión, analizando en ellas los tres elementos intervinientes. La elección de este periodo de años para la realización de la revisión teórica ha sido escogida para ofrecer una panorámica y un recorrido sobre la evolución que ha ido teniendo el tema tratado a lo largo de esos cinco años. Para ello se estudian, según Werbach y Hunter (2012), las dinámicas, las mecánicas y los componentes siguiendo una metodología cualitativa basada en el análisis de contenido. Los resultados indican que los procesos de gamificación en educación generan en los alumnos importantes beneficios, no exentos de dificultades en algunos de los trabajos del estudio. Tras esta argumentación, el artículo concluye a favor de beneficios de la gamificación en educación como la motivación, la inmersión para posibilitar la anticipación y planificación de situaciones; el compromiso y la socialización a través de la interactividad y la interacción; así como de la variedad de elementos que intervienen, lo que hace la actividad educativa más motivante y estimulante para los alumnos.

\section{Palabras clave}

Gamificación - Gamificación en educación - Motivación - Juego.

1- Universidad de Jaén, Jaén, España. Contactos: aortiz@ujaen.es; m.agredamontoro@gmail.com

2- City of London School, Londres, Reino Unido. Contacto: juanjordanortiz@gmail.com

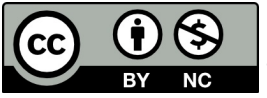




\section{Gamification in education: an overview on the state of the art}

\section{ABSTRACT}

The new society of knowledge and technology has created a new map in which young people feel concerns which education has not always been able to satisfy. This new scenario has changed the interests of students. Therefore, teachers need to explore new strategies and resources to increase the motivation and engagement of the students. The aim of this paper is to review the benefits of the use of gamification and its application in education. Several academic publications between 2011 and 2016 regarding to the use of gamification, motivation and immersion, have been examined. The choice of this period of time has been made in order to offer a review of the evolution and development of gamification during those five years. According to Werbach and Hunter (2012), it is necessary to study the game dynamics, mechanics and components under a qualitative methodology based on the analysis of the content. Results show that gamification processes in education generate important benefits to the students, not without some difficulties. The article concludes in support of the benefits of the use of gamification in education, such as motivation, immersion in order to plan new situations and anticipate them, the engagement and the sociability through interaction and interactivity, as well as the great number of different elements involved in the process, which make education more motivating and engaging for students.

\section{Key words}

Gamification - Gamification in education - Motivation - Game.

\section{Introducción}

Las tendencias e inquietudes actuales exigen cada día más una respuesta a las generaciones de jóvenes que necesitan encontrar respuesta en el contexto educativo a sus expectaciones tecnológicas y necesidades más inmediatas. Esto trae consigo la responsabilidad de profesores e instituciones a la hora de innovar en metodologías emergentes que intenten incorporar en sus clases estrategias que aumenten la motivación y el compromiso de proporcionar todas las herramientas y recursos posibles que favorezcan el aprendizaje autónomo y significativo de sus alumnos. Además, ha quedado constancia de que los estudiantes alcanza un gran nivel de compromiso cuando se encuentran motivados, incluso prefiriendo seguir con la actividad lúdica a dar por finalizada la clase (FERNÁNDEZ; OLMOS; ALEGRE, 2016). Dentro de estas nuevas demandas surgen nuevas estrategias docentes. En este artículo nos centramos en la gamificación en la educación o gamificación educativa (MARÍN, 2015). 
Esta tendencia, aparece recogida en el informe Horizon Report: $2014 \quad \mathrm{~K}-12$ (JOHNSON et al., 2014), cuyas recomendaciones se plantean en un plazo de dos a tres años en referencia al aprendizaje basado en juegos y la gamificación como estrategia didáctica, integrando aspectos de la dinámica del juego en contextos no lúdicos que ayuden a potenciar la motivación de los estudiantes, así como otros valores positivos que son usuales en la mayoría de los juegos que se utilizan actualmente para el aprendizaje.

En la mayor parte de las disciplinas los juegos están orientados al objetivo de aprendizaje teniendo fuertes componentes sociales y plantean simulaciones de algún tipo de experiencia del mundo real que los estudiantes encuentran relevante para sus vidas. Como se desprende del informe, la acogida del juego en el mundo académico está haciendo que los desarrolladores respondan con juegos expresamente diseñados para apoyar el aprendizaje inmersivo y experiencial.

El objetivo de este trabajo es hacer una revisión del impacto, los beneficios del uso de la gamificación y su aplicación en el contexto educativo. Para ello, se realiza una aproximación a la gamificación desde el juego en diferentes sectores (educación, empresas, recursos humanos, etc.), acercándonos a ámbitos muy diferentes como Nike, Volkswagen y su Fun Theory, Weight Watchers, Deloitte Touche Tohmatsu Ltda., entre otras. El centro de atención lo constituye la gamificación en educación, dada la importancia que están adquiriendo los beneficios que aporta un diseño curricular basado en los principios de la gamificación, ayudando a mantener el interés de los alumnos y evitando que el proceso de enseñanza-aprendizaje se convierta en algo aburrido o sin interés para ellos, disminuyendo el número de abandonos y la falta de compromiso en el proceso de enseñanza, favoreciendo la adquisición de competencias (AREA; GONZÁLEZ, 2015).

La revisión de la literatura se completa con un análisis documental de determinadas experiencias gamificadas, con el fin de estudiar los tres elementos intervinientes según Werbach y Hunter (2012), las dinámicas, las mecánicas y los componentes, aportando, en base a los resultados, conclusiones relevantes que vienen a confirmar los resultados planteados por los autores mencionados, y el uso que en las experiencias seleccionadas se hace de ellos.

En base a los planteamientos expuestos, este trabajo está guiado por las siguientes preguntas de investigación:

1. ¿Qué beneficios aporta el uso de la gamificación en educación y su aplicación en el contexto educativo?

2. ¿En qué medida se confirman los elementos intervientes en la gamificación según Werbach y Hunter (2012) en las investigaciones seleccionadas en el estudio?

\section{Marco teórico}

Desde hace años se han introducido aspectos lúdicos en todos los espacios de nuestra vida (ESCRIBANO, 2013). Así, se han producido intentos de gamificar actividades en sectores muy diferentes (educación, empresas, recursos humanos, etc.). En este sentido, encontramos ejemplos de gamificación en muy diferentes ámbitos: 
Nike y el ejercicio físico, mediante su aplicación Nike+, Volkswagen y su Fun Theory para combatir la seguridad vial, el sedentarismo o el cambio climático. En el mundo de las dietas, el programa Weight Watchers busca hacer de la pérdida de peso una actividad más divertida y llevadera. En el ámbito de las empresas, la consultora Deloitte Touche Tohmatsu Ltda. está incorporando elementos de los videojuegos en sus centros de trabajo. Están implementando tácticas de recompensas y competición que se suelen encontrar en el mundo de los juegos para hacer que tareas como la formación de directivos, la introducción de datos y las tormentas de ideas no parezcan tanto un trabajo. En el mundo empresarial, las empresas buscan aumentar el compromiso de los clientes y los trabajadores, de ahí la importancia de entender cuáles son los elementos que facilitan que un empleado se implique (ROBLEDO; NAVARRO; JIMÉNEZ, 2013). La gamificación favorece ese compromiso y es una herramienta fundamental para conseguir el llamado engagement.

La gamificación se basa en el uso de elementos del diseño de videojuegos en contextos que no son de juego para hacer que un producto, servicio o aplicación sea más divertido, atractivo y motivador (DETERDING, 2011). Por su parte Zichermann (2012), añade que mediante la introducción de mecánicas y planteamientos de los juegos, se busca involucrar a los usuarios. Así pues, Burke (2012) plantea la gamificación como el uso de diseños y técnicas propias de los juegos en contextos no lúdicos con el fin de desarrollar habilidades y comportamientos de desarrollo. En este contexto, nuestro planteamiento de gamificación hace referencia a la aplicación de mecánicas de juego a ámbitos que no son propiamente de juego, con el fin de estimular y motivar tanto la competencia como la cooperación entre jugadores (KAPP, 2012, 2016).

De forma mayoritaria, los autores coinciden en señalar la gamificación como un factor fundamental para aumentar la motivación de los usuarios. Motivar es despertar la pasión y el entusiasmo de las personas para contribuir con sus capacidades y talentos a la misión colectiva (DETERDING, 2011, 2012). Así pues, si se quieren utilizar técnicas de gamificación, se necesita conocer las claves de la motivación para diseñar juegos que enganchen a los distintos tipos de jugadores como veremos más adelante (VALDERRAMA, 2015). De este modo, las técnicas de gamificación están irrumpiendo con fuerza en las organizaciones con el fin de potenciar la motivación y compromiso de empleados y clientes. Los ámbitos de uso van desde la innovación, el marketing, la gestión del talento y el aprendizaje, hasta el desarrollo de hábitos saludables y responsables (VALDERRAMA, 2015).

En este contexto, los fundamentos de la gamificación según Werbach (2012), son las dinámicas, las mecánicas y los componentes. Las dinámicas son el concepto, la estructura implícita del juego. Las mecánicas son los procesos que provocan el desarrollo del juego y los componentes son las implementaciones específicas de las dinámicas y mecánicas: avatares, insignias, puntos colecciones, rankings, niveles, equipos, entre otros. La interacción de estos tres elementos es lo que genera la actividad gamificada como se presenta en la figura 1. 
Figura 1- Pirámide de los Elementos de gamificación

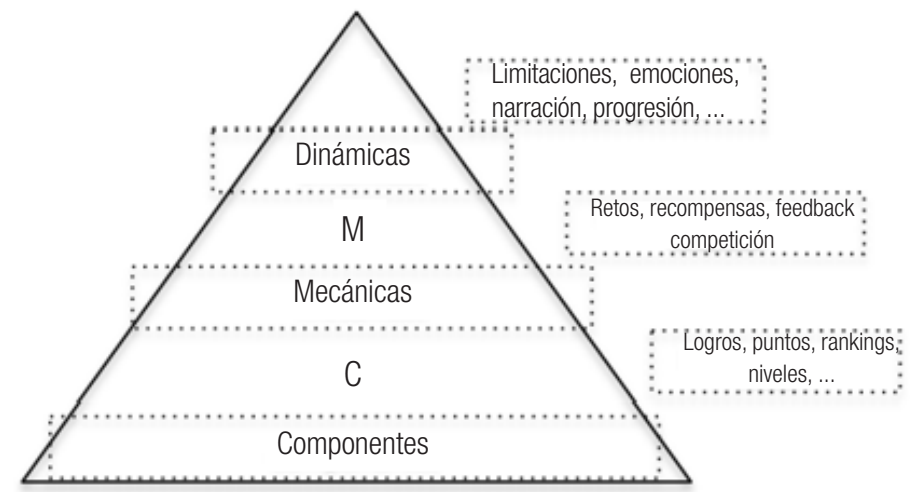

Fuente: Adaptado de Werbach (2012).

En el contexto educativo, la gamificación está siendo utilizada tanto como una herramienta de aprendizaje en diferentes áreas y asignaturas, como para el desarrollo de actitudes y comportamientos colaborativos y el estudio autónomo (CAPONETTO; EARP; OTT, 2014). De hecho, no debe verse tanto como un proceso institucional sino directamente relacionado con un proyecto didáctico contextualizado, con significatividad y transformador del proceso de enseñanza-aprendizaje (CAROLEI et al., 2016). Son numerosas las investigaciones que llegan a plantear beneficios con el uso de mecánicas de gamificación en el aula (GONZÁLEZ; BLANCO, 2008; FITZ-WALTER; TJONDRONEGORO; WYETH, 2011; BARATA et al., 2013; LI et al., 2013; GONZÁLEZ; MORA, 2014), llegando a plantear las propias actividades gamificadas como estrategias didácticas (AREA; GONZÁLEZ, 2015). Sin embargo, hay que tener en cuenta que desarrollar juegos específicos para los contextos educativos implica un elevado costo, ya que se intenta luchar contra la sobreestimulación a la que se enfrenta el alumnado por la cantidad de videojuegos comerciales a los que acceden. Además, si el alumnado no muestra interés alguno por su aprendizaje la gamificación de una actividad puede incluso llegar a contaminar el proceso de enseñanza-aprendizaje (TORI, 2016).

En relación al alumnado, Prensky (2005) plantea cómo lo que desea el alumno de hoy en día es ver que sus opiniones tienen valor, seguir sus propias pasiones e intereses, crear nuevas cosas utilizando todas las herramientas que les rodean, trabajar mediante proyectos en grupo, tomar decisiones y compartir control, cooperar y competir. Los alumnos necesitan sentir que la educación que reciben es real, que tiene valor.

De este modo, la gamificación puede favorecer todos estos deseos de los alumnos mediante las distintas mecánicas y dinámicas del juego, pero como señalan Castellón y Jaramillo (2012), es muy importante que haya una relación controlada entre los retos que se muestran a los alumnos y la capacidad de estos para llevarlos a cabo, pues si un reto es demasiado fácil, provocará aburrimiento en el alumno, mientras que un reto inalcanzable supondrá la frustración, concluyendo ambas opciones en una pérdida de motivación por el aprendizaje, siendo las recompensas un aspecto muy importante de la gamificación. Durante mucho tiempo las únicas recompensas que los alumnos han 
adquirido han sido las calificaciones, la gamificación hace más frecuente la obtención de recompensas. Salen y Zimmerman (2004) destacan que los ejercicios que se realizan mediante la gamificación deben presentar tres niveles: la creación del juego, la modificación del juego y el análisis de juego que deben estar impregnados de un diseño interactivo. Por tanto, el profesorado tiene la importante tarea de realizar un análisis y selección de aquellas actividades gamificadas que atiendan a los intereses y necesidades del alumnado dentro de la labor docente.

Así pues, un diseño curricular basado en los principios de la gamificación ayuda a mantener el interés de los alumnos evitando que el proceso de enseñanza-aprendizaje se convierta en algo aburrido o sin interés, en línea con Yunyongying (2014). La educación es un campo en el que la gamificación está viendo crecer su importancia. Scott y Neustaedter (2013) recogen cuatro conceptos fundamentales a la hora de entender la importancia y los beneficios de la gamificación: libertad para fallar, rápido feedback, progreso, historia.

Otro aspecto a considerar en la gamificación es la motivación: uno de los mayores retos a los que se enfrenta un docente de cara al aprendizaje. La motivación se demuestra mediante la elección personal de compromiso hacia una actividad y determina la intensidad del esfuerzo y persistencia en esa actividad (GARRIS; AHLERS; DRISKELL, 2002). Para Soriano (2001), la motivación es más un proceso dinámico que un estado fijo, los estados motivacionales de los seres humanos están en continuo flujo, tanto creciente como decreciente. Son dos los tipos de motivación que vamos a considerar en nuestro trabajo: la motivación intrínseca y la motivación extrínseca. La motivación extrínseca es provocada desde fuera del organismo. Este tipo de motivación es la que según Soriano (2001) se ha venido utilizando hace años en la educación: premiar a los alumnos mediante sus notas, comportamientos, insignias digitales (PALAZÓN-HERRERA, 2015).

Por el contrario, la motivación intrínseca es aquella que nace en el individuo y lo activa hacia aquello que le apetece, le interesa y le atrae. En esta línea, Valderrama (2015) señala el juego como una actividad intrínsecamente motivadora en la que nos implicamos por puro placer. Defiende que el juego nos permite crear situaciones de aprendizaje y experimentación para desarrollar habilidades de inteligencia emocional y social.

La escuela hoy en día presenta grandes problemas relacionados con el compromiso y la motivación de los alumnos, por lo que Lee y Hammer (2011) ven en la gamificación una oportunidad para solucionar estos problemas, al aprovechar el poder motivacional de los juegos en aspectos importantes del mundo real, favoreciendo la motivación del alumnado. La idea básica de la gamificación es el uso del poder de los juegos para otros propósitos (SAILER et al., 2013). Para ello, se recogen una serie de elementos mediante los cuales, la motivación se puede conseguir (puntos, badges, leaderboards, barras de progreso y avatares). Cada uno de estos elementos por separado intensifica algunos sentimientos como la capacidad, autonomía y las relaciones sociales. Pero en gamificación, todas estas sensaciones tienen a darse al mismo tiempo a través de los diferentes elementos del juego.

Uno de los principios que recogen Perrotta y otros autores (2013) para defender el uso del aprendizaje basado en el juego es el fomento de la motivación intrínseca que el juego posee, que llevada al campo de la educación ayuda al profesor a trabajar en un contexto de persuasión e invitación en lugar de obligación. 
Otra cuestión, algo más compleja, tiene que ver con el concepto de inmersión y su influencia en el proceso de aprendizaje y cómo dicha inmersión se puede favorecer a través de la gamificación (CASTELLÓN; JARAMILLO, 2012). Una experiencia se considera inmersiva cuando se quiere profundizar en ella, saber más y lograr un nivel de conocimiento más amplio. Un claro ejemplo son los videojuegos: en ellos el desbloqueo de nuevos escenarios, poderes, habilidades, generan la sensación de inmersión total. Por otro lado, presentan cómo en la educación tradicional no es posible esa sensación de inmersión pues toda la información se muestra dada por el profesor. Es por ello que Perrotta y otros autores (2013) recogen cómo la gamificación de la educación puede provocar dicha sensación mediante el aprendizaje a través del disfrute y la diversión.

La gamificación es una herramienta que puede convertir el aprendizaje en una actividad inmersiva. Perrotta y otros autores (2013) señalan que el hecho de aprender mediante disfrute y diversión puede ser un medio para introducir a los alumnos en un estado de flow. Este estado, traducido al español como flujo, refiere a la sensación de inmersión completa en una tarea.

Esta nueva concepción (CASTELLÓN; JARAMILLO, 2012) presenta una serie de cambios y desafíos: encontrar la pasión del estudiante, profundizar a veces más allá de los planes de estudio y que el profesor no tenga miedo a perder el control de la clase. Destacan que lo ideal es que el alumno supere al maestro. Csikszentmihalyi (1998) define el estado de flujo como el motor para el aprendizaje. Por tanto, una gamificación bien aplicada, en nuestra opinión, provocará un aumento de la motivación, el rendimiento y el aprendizaje en los alumnos, que a través de los elementos y principios del juego, mostrarán un mayor compromiso e interés por el aprendizaje, estando en ese estado de flujo.

El proyecto de gamificación Zombie-Based Learning (aprendizaje basado en zombies), llevado a cabo por Hunter (2017), crea una historia narrativa en la que los alumnos sufren una invasión de zombies de la que deben escapar. El profesor va guiando la historia, presentando nuevos escenarios, nuevas situaciones a las que los alumnos deben responder. La idea principal es que los alumnos sepan interpretar los datos y la simbología de los mapas y realizar relaciones espaciales entre lugares. El profesor guía el aprendizaje mediante las preguntas y retos que lanza, busca que los alumnos cumplan con los objetivos de conocimiento establecidos en el currículum. El resultado final del proyecto es un mapa elaborado por parte de los alumnos, en los que se recogen los datos y direcciones seguidas para escapar de dicha invasión. No sólo se abarca la temática de localización y distancias, sino también otros contenidos de la asignatura como las corrientes migratorias que pueden afectar a la expansión de los zombies, o las diferentes zonas climáticas. El currículo educativo está diseñado para ser flexible y para favorecer la creatividad y compromiso de alumnos y profesores. Con este proyecto, el objetivo fundamental del autor es ejercitar, entrenar y desarrollar las habilidades superiores y elevadas del pensamiento, llegar a pensar como un profesional de la geografía (BLANTON, 2012).

Como recogen Gallego et al. (2014) en la misma línea, la gamificación abre una nueva vía hacia al aprendizaje basado en proyectos. Este tipo de aprendizaje involucra al estudiante en un proyecto complejo y más cercano a la realidad, en línea con las necesidades que la sociedad demanda en la actualidad coincidiendo con las necesidades 
presentadas por Prensky (2005), en relación al papel activo del estudiante y el aprendizaje cooperativo.

\section{Metodología}

En este trabajo se adopta una metodología cualitativa basada en el análisis de contenido, seleccionando contenido relevante de publicaciones científicas encontradas en bases de datos y repositorios accesibles a la temática como EBSCOHost, Proquest, Web of Science, Scopus, ScienceDirect, Google Scholar, ACM Digital library, lo que nos ha permitido revisar una serie de experiencias gamificadas que mediante búsquedas selectivas cruzadas de documentos a partir de determinadas palabras clave relevantes (WERBACH; HUNTER, 2012) nos permitan revisar el estado de la cuestión sobre el tema.

\section{Procedimiento}

Los elementos que se han considerado en el análisis hacen referencia a la gamificación en educación, la motivación e inmersión. Dichos elementos se han cruzado con otros considerados clave, como son: componentes, mecánicas, y dinámicas, que facilitarán la creación de la línea narrativa de los contenidos (WERBACH; HUNTER, 2012), encontrando en un total de 330 documentos durante los años 2011-2016, de los que se han seleccionado 37 que se ajustaban a las exigencias planteadas en el estudio, con una selección final de cinco para posibilitar un análisis en profundidad de cada una de esas experiencias educativas gamificadas.

\section{Resultados}

Se han analizado 37 experiencias de gamificación llevadas a cabo en diferentes contextos en los últimos seis años, cada una de ellas con diferentes características, cuyo eje común es el beneficio de su aplicación en educación, la motivación de los alumnos en el aprendizaje y la incorporación de elementos propios del juego en la actividad educativa. Para ello se presenta por un lado, un catálogo de los cinco estudios seleccionados, y por otro un análisis de los mismos, siguiendo las tres categorías de elementos de juego definidas por Werbach y Hunter (2012).

\section{Catálogo de estudios gamificados}

Los estudios seleccionados engloban experiencias en contextos escolares y universitarios con el fin de conocer la existencia de elementos diferentes en cada uno de los estudios.

\section{Minecraft Edu.}

Sáez y Domínguez (2014) aplican a un grupo de 41 alumnos una metodología basada en la aplicación Minecraft Edu. El tema a tratar serán los edificios históricos. Este grupo de alumnos asistieron a varias sesiones dedicadas a esta temática usando 
dicha herramienta. Una vez finalizado el proceso, se compararon sus resultados con los de otros alumnos de la misma edad, que habían recibido sesiones de la misma temática pero con una metodología más tradicional. Los resultados fueron mejores en aquellos alumnos que usaron Minecraft $E d u$, si bien las diferencias no fueron significativas. Según señalan los autores todos los alumnos del grupo de gamificación expresaron que las clases se convirtieron en más dinámicas y divertidas y que se habían sentido protagonistas activos del proceso. Los autores también recogen algunas valoraciones negativas por parte de padres a la hora de usar esta herramienta, pues la veían como una pérdida de tiempo. Pero en general, la mayor parte reconoce que este enfoque mejora la creatividad, desarrolla el descubrimiento, es divertido y aporta ventajas interactivas.

2. Motivación del alumnado de educación secundaria a través del uso de insignias digitales.

Palazón-Herrera (2015) desarrolla un estudio cuantitativo no experimental con 54 alumnos, basado en insignias digitales como sistema de acreditación de aprendizajes. Está centrado en el modelo socio-cognitivo propuesto por Pintrich (1998), en el que las insignias pueden ser un elemento muy motivador orientado a la consecución de metas educativas, constituyendo igualmente un elemento motivador a nivel afectivo. Por otro lado, la utilización de insignias ha provocado en el alumno un espíritu competitivo en el desarrollo de los proyectos, siendo muy motivador las metas relacionadas con la obtención de recompensas.

3. Estrategias para optimizar el aprendizaje y la adquisición de competencias en contextos universitarios.

Las autoras (VILLATUSTRE; DEL MORAL, 2015) plantean un juego de simulación social de carácter cuantitativo con 161 alumnos universitarios. Los estudiantes debian elaborar colaborativamente un plan de intervención capaz de promover el desarrollo sostenible en un contexto rural. La utilización de mecánicas de juego (misión, reglas, puntos, desafíos) al servicio del aprendizaje incrementó su motivación y nivel de satisfacción con la realización del proyecto solicitado. La gamificación ha favorecido el aprendizaje colaborativo en el desarrollo de la experiencia, independientemente de las herramientas digitales utilizadas.

4. Resortes de gamificación en aplicaciones de TVE.

La autora (BARRIENTOS, 2016) analiza los mecanismos de juego presentes en apps vinculadas a TVE que tienen diferentes usos de segunda pantalla. Emplea las tres categorias de elementos de juego definidas por Werbach y Hunter (2012): dinámicas, mecánicas y componentes. A partir de ellos, construye una matriz para el análisis con nueve aplicaciones de segunda pantalla: Rtve.es | Móvil, +TVE, +24, Clan, El tiempo en Rtve.es, Cuéntame cómo pasó, Master Chef, Saber y ganar y Los misterios de Laura. Los resultados del estudio de caso permiten conocer elementos de gamificación en todas las aplicaciones como es la dinámica de las emociones independientemente de su género y contenidos; relaciones de interacción en redes sociales; emerge como rasgo habitual la competición, ligada a concursos y premios, como los elementos más destacados. 
5. La competición como mecánica de gamificación en el aula: Una experiencia aplicando aprendizaje basado en problemas y aprendizaje cooperativo.

El estudio desarrollado por Cantador (2016) está basado en lecciones aprendidas en experiencias previas, utilizando la competición como una de las principales mecánicas de gamificación. Pretende conocer a través del aprendizaje basado en problemas y del aprendizaje cooperativo, los principios de competición saludable y las mecánicas de gamificación, y en qué medida dicha metodología incrementa la motivación de los estudiantes. La competición se desarrolló con sesenta estudiantes del grado de Ingeniería Química en la asignatura Informática aplicada, distribuidos en diez equipos de seis miembros. Los resultados obtenidos indican que un 75\% obtuvo una motivación alta en la competición así como una alta satisfacción en la utilidad de la actividad en su proceso de aprendizaje. Finalmente destaca la valoración positiva por parte de todos los estudiantes, del espíritu competitivo y cooperativo de la actividad.

En la tabla adjunta se presentan las características generales de los estudios seleccionados, en base a los elementos implementados (Dinámicas, Mecánicas y Componentes) según Werbach y Hunter (2012) en los cinco documentos del estudio, así como la categoría motivacional, el tipo de estudio, método implementado y muestra de estudio, siguiendo a Hamari, Koivisto y Sarsa, (2014).

Tabla 1- Características generales del análisis

\begin{tabular}{|c|c|c|c|c|c|c|c|c|}
\hline ESTUDIO & ACTIVIDAD & $\begin{array}{c}\text { CATEGORIA } \\
\text { MOTIVACIONAL }\end{array}$ & DINÁMICAS & MECÁNICAS & COMPONENTES & TIPO ESTUDIO & MÉTODO & N \\
\hline 1 & Minecraf-Edu & $\begin{array}{l}\text { Actitudes- } \\
\text { Interacción }\end{array}$ & $\begin{array}{c}\text { Juegos Serios } \\
\text { Narrativas }\end{array}$ & Creatividad & & Cuasiexperimental: & Mixto & 41 \\
\hline 2 & $\begin{array}{l}\text { Insignias } \\
\text { digitales }\end{array}$ & Socio-cognitivo & Proceso insignias & Metas educativas & Insignias digitales & Cuantitativo & No experimental & 54 \\
\hline 3 & $\begin{array}{l}\text { Juego de } \\
\text { simulación } \\
\text { social }\end{array}$ & Colaboración & $\begin{array}{l}\text { Logro competición } \\
\text { expresión }\end{array}$ & $\begin{array}{l}\text { Misión } \\
\text { reglas } \\
\text { puntos } \\
\text { desafíos }\end{array}$ & $\begin{array}{l}\text { Videojuego } \\
\text { Los Sims }\end{array}$ & Cuantitativo & $\begin{array}{l}\text { Implementación } \\
\text { experiencia }\end{array}$ & 161 \\
\hline 4 & APPs TV & Emociones & $\begin{array}{l}\text { Narrativas } \\
\text { trasmedia }\end{array}$ & $\begin{array}{l}\text { Logros } \\
\text { Objetivos }\end{array}$ & $\begin{array}{l}\text { Concursos } \\
\text { premios }\end{array}$ & Cualitativo & Estudio de casos: & $7^{*}$ \\
\hline 5 & $\begin{array}{l}\text { Competición } \\
\text { por equipos }\end{array}$ & Colaboración & $\begin{array}{l}\text { Proceso } \\
\text { socialización }\end{array}$ & $\begin{array}{l}\text { Competición } \\
\text { Puntuaciones } \\
\text { Ranking }\end{array}$ & Premios sorpresa & Cualitativo & Estudio de caso & 60 \\
\hline
\end{tabular}

* Número de casos

Fuente: Adaptado de Hamari, Koivisto y Sarsa, (2014).

Las categorías del estudio se han concretado en ocho: objetivo de la actividad, categoría motivacional, dinámicas, mecánicas, componentes, tipo de estudio, métodos utilizados y número de sujetos del estudio, aportando aspectos claves para el análisis de las experiencias seleccionadas. 


\section{Análisis de experiencias gamificadas}

El análisis de los elementos de gamificación utilizados en las cinco experiencias estudiadas dentro de las dinámicas, mecánicas y componentes, se presenta en la tabla adjunta, señalando los elementos de las mismas (BARRIENTOS, 2016).

Tabla 2- Matriz de análisis de componentes de gamificación

\begin{tabular}{|c|c|c|c|c|c|c|}
\hline \multirow{15}{*}{ Dinámica } & & 1. & 2. & 3. & 4. & 5. \\
\hline & Narrativa & $x$ & & & $x$ & \\
\hline & Progresión & & $x$ & $x$ & & $x$ \\
\hline & Restricciones & & & & & \\
\hline & Emociones & & $x$ & & $x$ & \\
\hline & Interacción social & $x$ & & $x$ & $x$ & \\
\hline & Desafíos & & & & & \\
\hline & Elementos aleatorios & & & & & \\
\hline & Competición & & $x$ & & & $x$ \\
\hline & Feedback & & & & & \\
\hline & Recompensas & & $x$ & & & $x$ \\
\hline & Obtención recursos & & & & $x$ & \\
\hline & Transacciones & & & & & \\
\hline & Turnos & & & & & \\
\hline & Cooperación jugadores & $x$ & & $x$ & & $x$ \\
\hline \multirow{8}{*}{ Mecánica } & Logros & $x$ & $x$ & $x$ & $x$ & $x$ \\
\hline & Avatares & & & & & \\
\hline & Insignias & & $x$ & & & \\
\hline & Desafíos & & & $x$ & & \\
\hline & Colecc. logros insignias & & $x$ & & & $x$ \\
\hline & Combate & & & & & \\
\hline & Cont. desbloqueado & & & & & \\
\hline & Regalos & & & & $x$ & $x$ \\
\hline \multirow{7}{*}{ Componentes } & Clasificaciones & & & & & $x$ \\
\hline & Niveles & & & & & $x$ \\
\hline & Puntos & & & $x$ & & $x$ \\
\hline & Búsquedas/aventuras & & & & & \\
\hline & Gráficas sociales & & & & & $x$ \\
\hline & Equipos & $x$ & & $x$ & & $x$ \\
\hline & Regalos virtuales & & & & & $x$ \\
\hline
\end{tabular}

Fuente: Elaboración propia adaptado de Barrientos (2016).

La experiencia Minecraft Edu (SÁEZ; DOMÍNGUEZ, 2014) utiliza como dinámicas las narrativas y las relaciones de interacción social, aplicando entre las mecánicas la cooperación entre los jugadores. Los componentes están basados en los logros y la formación de equipos. 
En cuanto al artículo sobre Motivación del alumnado de educación secundaria a través del uso de insignias digitales (PALAZÓN-HERRERA, 2015) incluye como dinámicas las emociones y la progresión; las mecánicas implementadas son la competición y las recompensas, siendo los componentes los logros y las insignias.

El trabajo desarrollado por Villatustre y Del Moral (2015), Estrategias para optimizar el aprendizaje y la adquisición de competencias en contextos universitarios, utiliza como componentes de las dinámicas la progresión y las relaciones de interacción social. En relación a las mecánicas, utiliza la cooperación entre jugadores, siendo los componentes utilizados logros, desafíos, puntos y trabajo por equipos.

El trabajo de Barrientos (2016), Resortes de gamificación en aplicaciones de TVE, incorpora como dinámicas la narrativa, las emociones y las relaciones de interacción social. En cuanto a las mecánicas incorpora la obtención de recursos, destacando los regalos y logros, entre los componentes.

El estudio sobre La competición como mecánica de gamificación en el aula: una experiencia aplicando aprendizaje basado en problemas y aprendizaje cooperativo, llevado a cabo por Cantador (2016), establece la progresión entre las dinámicas. Los componentes de las mecánicas son la competición, la cooperación entre jugadores y las recompensas. Los componentes utilizados son logros, colección de logros e insignias, regalos, clasificaciones, niveles, puntos, gráficas sociales, equipos y regalos virtuales.

Los resultados en referencia a los beneficios del uso de la gamificación en educación y su aplicación en el contexto educativo a efectos de motivación e inmersión hacen referencia a la mejora de la colaboración en el aula, las emociones como elemento favorecedor del proceso de enseñanza/aprendizaje, las actitudes e interacción y la cooperación entre compañeros.

Los resultados obtenidos en cuanto a los elementos intervinientes en la gamificación según Werbach y Hunter (2012) indican la utilización en distintos grados de los componentes estudiados, encontrando en los cinco estudios resultados diferentes.

En cuanto a los componentes, los logros y el trabajo por equipos aparecen en tres de ellos. En el caso de las dinámicas, tres de los estudios incorporan en sus dinámicas la progresión y las relaciones de interacción social; en cuanto a las mecánicas, tres de los estudios incorporan la cooperación entre jugadores. Por otro lado, las emociones son incorporadas como dinámicas en dos de los estudios.

\section{Discusión y conclusiones}

En este trabajo hemos establecido los principios y beneficios que la gamificación presenta en diferentes ámbitos, así como algunos de los riesgos y factores a tener en cuenta a la hora de gamificar. Con respecto a la producción científica de los últimos cinco años (2011-2015) relacionada con la gamificación, el análisis de los trabajos revisados ha permitido conocer los resultados de investigaciones realizadas, aportando y contrastando nuestro marco teórico. Por otro lado, los resultados de la matriz del análisis vienen a confirmar los elementos de la gamificación planteados por Werbach y Hunter (2012), así 
como los componentes incorporados en cada uno de ellos, en línea con los planteados por Barrientos (2016).

Con carácter más general, el estudio de la gamificación en contextos educativos nos acerca a conocer los beneficios de la gamificación en cuanto al peso de la motivación en el desarrollo de los estudios analizados. Los resultados nos han acercado a conocer el tipo de motivación que se plantea en las diferentes propuestas gamificadas, lo que hace que los alumnos perciban la experiencia como motivante en beneficio de su compromiso y aprendizaje. Podemos concluir de este modo, la gran influencia que tiene la gamificación en el desarrollo cognitivo de los estudiantes, en las emociones y en los procesos de socialización que se generan a lo largo del proceso.

La gamificación puede hacer de la educación una actividad inmersiva, que provoque en los alumnos una sensación de dedicación absoluta (PERROTA et al., 2013). Podemos considerar que gamificar es una actividad más compleja que aplicar un juego. Es necesaria una profunda reflexión sobre los objetivos que se quieren alcanzar: una vez determinados, se establecerán las normas que regirán el proceso. Por lo que llevar a cabo un proyecto de gamificación requiere una profunda planificación y puede encontrarse en ocasiones con resistencias a su implantación. Coincidiendo con Burke (2012), consideramos que la gamificación será una herramienta fundamental en el futuro, ya que su papel de crear compromiso facilitará la puesta en común y desarrollo de ideas nuevas, favoreciendo innovaciones en el aula, más allá de experimentos novedosos.

Entre los hallazgos del estudio destacan los niveles de motivación alcanzados, así como la implicación del profesorado en el diseño de las actividades gamificadas, en las diferentes publicaciones analizadas. Para finalizar, cabe señalar la necesidad de ampliar la muestra del análisis siendo cautos en los resultados obtenidos.

\section{Referencias}

AREA, Manuel; GONZÁLEZ, Carina. De la enseñanza con libros de texto al aprendizaje en espacios online gamificados. Educatio Siglo XXI, Murcia, v. 33, n. 3, p. 15-38, 2015. Disponible en: <http://dx.doi. org/10.6018/j/240791>. Acceso en: 28 jun. 2016.

BARATA, Gabriel et al. Engaging engineering students with gamification. In: INTERNATIONAL CONFERENCE ON GAMES AND VIRTUAL WORLDS FOR SERIOUS APPLICATIONS (VS-GAMES) ON IEE XPLORE, 5., 2013. Poole. v. 9, Fifth international... Poole: Bournemouth University, 2013. p. 1-8. Disponible en: <http:// dx.doi.org/10.1109/vS-GAMES.2013.6624228>. Acceso en: 3 en. 2017.

BARRIENTOS, Mónica. Resortes de gamificación en aplicaciones de TVE. Opción, Maracaibo, v. 32, n. 7, p. 178-195, 2016.

BLANTON, Robert. Zombies and international relations: a simple guide for bringing the undead into your classroom. International Studies Perspectives, v. 14, n. 1, p. 1-13, 2012. Disponoble en: <doi: 10.1111/ j.1528-3585.2012.00505.x>. Acceso en: 3 en. 2017. 
BURKE, Brian. Gamification 2020: what is the future of gamification? Standford: Gartnet, 2012.

CANTADOR, Ivan. La competición como mecánica de gamificación en el aula: una experiencia aplicando aprendizaje basado en problemas y aprendizaje cooperativo. In: CONTRERAS ESPINOSA, Ruth; EGUIA, José Luis (Ed.). Gamificación en aulas universitarias. Bellaterra: Institut de la Comunicació: Universitat Autònoma de Barcelona, 2016. p. 68-97.

CAPONETTO, Ilaria; EARP, Jeffrey; OTT, Michela. Gamification and education: a literature review. In: EUROPEAN CONFERENCE ON GAMES-BASED LEARNING, 8., 2014, Berlín. Actas... Berlín: University of Applied Sciencies, 2014. p. 50-57.

CAROLEl, Paula et al. Gamificação como elemento de uma política pública de formação de professores: vivências mais imersivas e investigativas. In: SIMPÓSIO BRASILEIRO DE GAMES E ENTRETENIMENTO DIGITAL (SBGames), 15., 2016, São Paulo. Atas... São Paulo: SBC: Escola Politécnica da USP, 2016. p. 1253-1256.

CASTELLÓN, Lucía; JARAMILLO, Óscar. Educación y videojuegos: hacia un aprendizaje inmersito. Homo Videoludens, Barcelona, v. 2, p. 264-281, 2012.

CSIKSZENTMIHALYI, Mihaly. Aprender a fluir. Barcelona: Kairós, 1998.

DETERDING, Sebastián. Gamification: designing for motivation. Interactions, New York, v. 19, n. 4, p. 14-17, 2012.

DETERDING, Sebastián. Gamification: toward a definition. In: TAN, Desney; BEGOLE, Bo (Ed.). Design, ACM CHI 2011.Vancouver: [s. n.], 2011. p. 12-15. Disponible en: <http://gamification-research.org/wp-content/ uploads/2011/04/02-Deterding-Khaled-Nacke-Dixon.pdf>. Acceso en: 10 mzo. 2017.

ESCRIBANO, Flavio. Gamificación versus Ludictadura. Obra Digital, Barcelona, n. 5, p.58-72, 2013.

FERNÁNDEZ, Anabel; OLMOS, Julia; ALEGRE, Joaquín. Pedagogical value of a common knowledge repository for business management courses. @ Tic: Revista d'Innovació Educativa, Valencia, n. 16, p. 39-47, 2016.

FITZ-WALTER Zachary; TJONDRONEGORO Dian; WYETH Peta. Orientation passport: using gamification to engage university students. In: AUSTRALIAN COMPUTER HUMAN-INTERACTION CONFERENCE, 23., 2011, Canberra. Actas... Canberra: Australian National University, 2011. p. 122-125.

GALLEGO, Francisco et al. Panorámica: serious games, gamification y mucho más. ReVisión, Castellón, v. 7, n. 2, p. 13-23, 2014.

GARRIS, Rosemary; AHLERS, Robert; DRISKELL, James. Games, motivation, and learning: a research and practice model. Simulation \& Gaming, London, v. 33, n. 4, p. 441-467, 2002. 
GONZÁLEZ Carina; BLANCO Francisco. Integrating an educational 3D game in moodle. Simulation \& Gaming, London, v. 39, n. 3, p. 399-413, 2008.

GONZÁLEZ Carina; MORA Alberto. Methodological proposal for gamification in the computer engineering teaching. In: SIERRA, José Luis; DODERO, Juan Manuel; BURGOS, Daniel (Ed.); INTERNATIONAL SYMPOSIUM, 2014, Logroño. Proceeding computers in education (SIIE). Logroño: Universidad de La Rioja, 2014. p. 29-34.

HAMARI, Juho; KOIVISTO, Jonna; SARSA, Harri. Does gamification work? A literature review of empirical studies on gamification. In: HAWAII INTERNATIONAL CONFERENCE ON SYSTEM SCIENCE, 47., 2014, Manoa. Actas... Manoa: University of Hawaii, 2014. p. 3025-3034.

HUNTER, David. Zombie-Based Learning: geography taught in zombie apocalypse- kickstarter. 2017. Disponible en: <https://www.kickstarter.com/projects/hunterd/dead-reckon-the- zombie-based-learninggraphic-nove>. Acceso en: 30 jun. 2017.

JOHNSON, Larry et al. NMC Horizon Report 2014: higher education edition. Austin: The New Media Consortium, 2014.

KAPP, Karl. Games, Gamification, and the quest for learner engagement. Training and Development, Reino Unido, v. 66, n. 6, p. 64-68, 2012.

KAPP, Karl; LATHAM, William; FORD-LATHAM, Hester. Integrated learning for ERP success: a learning requirements planning approach. Florida: CRC Press, 2016.

LEE, Joey; HAMMER, Jessica. Gamification in education: what, how, why bother? Academic Exchange Quarterly, New York, v. 15, n. 2, p. 146-151, 2011.

LI, Cen, et al. Engaging computer science students through gamification in an online social network based collaborative learning environment. International Journal of Information and Education Technology, v. 3, n. 1, p. 72-77, 2013.

MARÍN, Verónica. La gamificación educative: una alternativa para la enseñanza creativa. Digital Education Journal, Barcelona, n. 90, p. 1-4, 2015. Disponible en: <http://revistes.ub.edu/index.php/der/article/ view/13433/pdf>. Acceso en: 15 my. 2016.

PALAZÓN-HERRERA, José. Motivación del alumnado de educación secundaria a través del uso de insignias digitales. Opción, Maracaibo, n. 31, p. 1059-1079, 2015.

PERROTTA, Carlo et al. Game-based learning: latest evidence and future directions. NFER Research Programme, 2013. p. 1-35. Disponible en: <http://www.nodo-observa.es/sites/default/files/GAME01.pdf>. Acceso en: 20 febr. 2017. 
PINTRICH, Paul. A process-oriented view of student motivation and cognition. In: STARK, Joan; METS, Lisa (Ed.). Improving teaching and learning through research: new directions for institutional research. San Francisco: Jossey-Bass, 1998. p. 65-79.

PRENSKY, Marc. Listen to the natives. Educational Leadership, Vancouver, v. 63, n. 4, p. 8-13, 2005.

ROBLEDO, José Luis; NAVARRO, Fernando; JIMÉNEZ, Sergio. Gamificación como estrategia de marketing interno. Intangible Capital, Barcelona, v. 9, n. 4, 2013. Disponible en: <http://dx.doi.org/10.3926/ic.455>. Acceso en: 23 mzo. 2017.

SÁEZ, José Manuel; DOMÍNGUEZ, Concepción. Integración pedagógica de la aplicación minecraft edu en educación primaria: un estudio de caso. Revista de Medios y Educación, Sevilla, n. 45, p. 95-110, 2014.

SAlLER, Michael et al. Psychological perspectives on motivation through gamification. Interaction Design and Architecture Journal, Roma, n. 19, p. 28-37, 2013.

SALEN, Katie; ZIMMERMAN, Eric. Rules of play: game design fundamentals. Massachusetts: MIT Press, 2004.

SORIANO, Mariano. La motivación, pilar básico de todo tipo de esfuerzo. Proyecto Social, Zaragoza, n. 9, p. 163-184, 2001.

STOTT, Andrew; NEUSTAEDTER, Carman. Analysis of gamification in education. Surrey: Connections Lab: Simon Fraser University, 2013.

TORI, Romero. Tecnologia e metodologia para uma educação sem distância. EaD EmRede, Porto Alegre, v. 2, n. 2, p. 44-55, 2016.

VALDERRAMA, Beatriz. Los secretos de la gamificación: 10 motivos para jugar. Capital Humano, Madrid, $\mathrm{n}$. 295, p. 73-78, 2015.

VILLALUSTRE, Lourdes; DEL MORAL, María Ester. Gamificación: estrategia para optimizar el proceso de aprendizaje y la adquisición de competencias en contextos universitarios. Digital Education Review, Barcelona, n. 27, p. 13-31, jun. 2015. Disponible en: <http://greav.ub.edu/der/>. Acceso en: 1 abr. 2017.

WERBACH, Kevin. For the win: how game thinking can revolutionize your business. Wharton: Wharton Digital Press, 2012.

WERBACH, Kevin; HUNTER, Dan. For the win: how game thin-king can revolutionize your business. Philadelphia: Wharton Digital Press, 2012.

YUNYONGYING, Pete. Gamification: implications for curricular design. Journal of Graduate Medical Education, Polonia, v. 6, n. 3, p. 410-412, 2014. 
ZICHERMANN, Gabe. Rethinking elections with gamification: huffington post, nov. 2012. Disponible en: $<$ https://www.huffingtonpost.com/gabe-zichermann/improve-voter-turn-out_b_2127459.html>. Acceso en: 10 jun. 2017.

Recibido en: 21.12.2016

Revisiones en: 23.06.2017

Aprobado en: 08.08.2017

Ana-M Ortiz-Colón es doctora en Psicopedagogía por la Universidad de Jaén (España). Profesora titular de la Universidad de Jaén en el área de Didáctica y Organización escolar del departamento de Pedagogía.

Juan Jordán es graduado en Ciencias de la Actividad Física y del Deporte por la Universidad de Extremadura. Actualmente desarrolla su actividad docente como profesor de Educación Secundaria en el City of London School, United Kingdom.

Míriam Agreda es doctora en Ciencias de la Educación por la Universidad de Granada y diplomada en Educación Especial por la Universidad de Córdoba. Desarrolla su actividad docente e investigadora en la Universidad de Jaén, España. 